\title{
1 Exposition aux perturbateurs endocriniens et développement des maladies
}

\section{allergiques}

Exposure to Endocrine Disruptors and Development of Allergic Diseases

Laurence Guzylack-Piriou $^{1}$ et Grégory Bouchaud ${ }^{2}$

${ }^{1}$ Equipe de Neuro-Gastroentérologie and Nutrition, Toxalim (Centre de recherche en toxicologie alimentaire), Université de Toulouse, INRA, ENVT, INP-Purpan, UPS, Toulouse, France.

${ }^{2}$ UR 1268 BIA, Inra, 44300 Nantes, France

Résumé: La prévalence des maladies allergiques augmente dans le monde entier avec une complexité et une sévérité sans précédent. Les allergies les plus courantes chez les enfants sont les allergies alimentaires (8\%), l'eczéma (10\%) et l'asthme (10\%). Les causes précises de cette augmentation ne sont pas entièrement connues. Notamment, l'exposition humaine a des polluants environnementaux tels que les perturbateurs endocriniens (PE) a attiré l'attention ces dernières années. Cette revue met en lumière des recherches récentes explorant les effets de l'exposition aux PE dans les maladies allergiques. Parmi ces PE, le bisphénol A, les phthalates, le triclosan et le parabène ont démontré des effets sur le développement des allergies. Des études épidémiologiques ont montré qu'ils peuvent agir directement sur le système immunitaire en entrainant une rupture de la tolérance ou indirectement via la modulation du microbiote. De plus, les hormones sexuelles, également dérégulées par l'exposition aux $\mathrm{PE}$, favorisent la sensibilisation allergique dans des modèles animaux et peuvent être à l'origine du développement de troubles atopiques chez l'humain. Ces données émergentes sur le développement d'allergie après une exposition alimentaire et par inhalation à ces substances chimiques démontrent l'intérêt fondamental d'en comprendre les mécanismes et d'en prévenir les risques.

Mots clés : Allergie ; période périnatale ; perturbateurs endocriniens ; modèle animal; immunité 
Abstract: The prevalence of allergic diseases is increasing worldwide with unprecedented complexity and severity. The most common allergies in children are food allergies $(8 \%)$, eczema $(10 \%)$ and asthma (10\%). The exact causes of this increase are not fully understood. Notably, human exposure to environmental pollutants such as endocrine disruptors (EDs) has attracted attention in recent years. This review highlights recent research exploring the effects of ED exposure in allergic diseases. Among these EDs, bisphenol A, phthalates, triclosan and paraben have demonstrated harmful effects in terms of the development of allergies. Epidemiological studies have shown that they may act either directly on the immune system leading to disturbance of tolerance or indirectly via modulation of the microbiota. In addition, sex hormones, which are also deregulated by ED exposure, promote allergic sensitization in animal models and may cause atopic disorders in humans. These emerging data on the development of allergy following dietary and respiratory exposure to such chemicals demonstrate the critical value of understanding their mechanisms and preventing the associated risks.

Keywords: Allergies; childhood; endocrine disruptors; mouse model; immunity.

(1)

2

3

4

5

\section{.}


Introduction

Les maladies allergiques, telles que les allergies respiratoires, cutanées et alimentaires, ont considérablement augmenté au cours des dernières décennies. Ces maladies coexistent généralement et sont fréquentes dans les populations pédiatriques à l'échelle mondiale. Les enfants atteints d'une maladie allergique développent fréquemment d'autres maladies allergiques. La séquence de progression de la maladie est appelée "marche atopique" (1). Par exemple, les nourrissons avec l'eczéma sont plus à risque de développer une allergie alimentaire, les enfants allergiques aux œufs ont de fort risque de développer des maladies allergiques respiratoires, et les enfants atteints de rhino-conjonctivite allergique ont un risque accru de développer de l'asthme (2). Les réactions indésirables aux aliments sont extrêmement fréquentes et généralement attribués à l'allergie. Cependant, des manifestations cliniques de divers degrés de gravité liés à l'ingestion d'aliments peuvent survenir à la suite d'un certain nombre de troubles, dont certains seulement peuvent être définis comme allergiques, impliquant un mécanisme immunitaire. Les réactions alimentaires peuvent avoir une physiopathologie à médiation IgE, non-IgE ou une combinaison des deux impliquant la peau, le tractus gastro-intestinal, les voies respiratoires et/ou le système cardiovasculaire. L'allergie alimentaire résulte d'un manque de tolérance orale, un état de non-réponse systémique à un antigène soluble médié entre autres par les cellules T régulatrices dans le tractus gastro-intestinal. Le tissu lymphoïde intestinal associé à l'intestin est le plus grand organe lymphoïde secondaire dans le corps humain. Les cellules de ces tissus prélèvent constamment des antigènes étrangers ingérés. Ce tissus a évolué pour être capable de discriminer les agents pathogènes, potentiellement dangereux et les antigènes non nuisibles. Comme exemple de l'importance de la tolérance orale, l'induction d'une réponse immunitaire à médiation IgE chez la souris est très difficile après l'immunisation parentérale avec des antigènes tels que l'ovalbumine, les protéines du lait de vache ou d'arachide présent dans le régime. La tolérance peut être transférée 
aux animaux naïfs par le transfert de cellules $T$ régulatrices, qui sont des cellules clés dans la tolérance orale. La tolérance orale est un défaut phénoménalement efficace de réponse aux antigènes alimentaires ingérés, comme démontré par le fait que la plupart des humains n'ont aucune allergie alimentaire malgré qu'ils peuvent ingérer plusieurs tonnes de nourriture pendant sa vie. Cependant, lorsque la tolérance orale échoue, une allergie alimentaire se développe. L’allergie alimentaire est plus fréquente chez les nourrissons et les jeunes enfants, en raison de l'immaturité de la barrière intestinale et de leur système immunitaire $(3,4)$. En effet, un régime alimentaire approprié au cours de la petite enfance favorise la tolérance alimentaire (5).

Habituellement, les aliments, comme d'autres substances étrangères telles que le pollen, les poils de chat, etc., sont bien tolérés. Mais pour une raison qui reste encore mal élucidée, en leur présence, notre organisme réagit parfois en produisant des anticorps particuliers, les immunoglobulines $E$, ou IgE, responsables des symptômes de l'allergie : urticaire, boutons, crises d'asthme, etc... Dans les faits, les allergies alimentaires ont doublé en cinq ans et touchent aujourd'hui au moins $4 \%$ des Français adultes et $8 \%$ des enfants (6). Tous les aliments peuvent en principe provoquer une allergie, mais le lait, l'œuf et l'arachide concernent $80 \%$ des allergies des enfants. De même, les allergies respiratoires touchent $10 \%$ à $15 \%$ de la population avec un nombre de cas en augmentation constante (7). D'où vient cette hypersensibilité croissante? Les causes sont multiples. Des études épidémiologiques ont identifiées une variété de facteurs de risques dans le développement des maladies allergiques. Les expositions aux produits chimiques présents dans l'environnement sont des modulateurs potentiels des réponses allergiques (8, 9). Parmi eux, l'exposition aux perturbateurs endocriniens (PE) semble jouer un rôle important dans le déclenchement et l'exacerbation des maladies allergiques (10).

Les perturbateurs endocriniens sont des composés naturels ou chimiques ayant la capacité d'altérer les fonctions endocrines dans le corps en mimant ou bloquant les hormones endogènes (11). En 2012, I'OMS les définit comme "substances chimiques d'origine naturelle ou artificielle, étrangères à l'organisme qui peuvent interférer avec le fonctionnement du système endocrinien et 
induire ainsi des effets délétères sur cet organisme chez un individu ou chez ces descendants ». Cette définition a été reprise par l'Union européenne. Ces molécules agissent à très faibles doses (comparables aux concentrations physiologiques des hormones). Les interactions synergiques entre perturbateurs et des effets dose-dépendant non linéaires remettent en question les approches réglementaires basées sur des « seuils toxicologiques » par produit (12). Cette hypothèse en faveur d'une association entre l'exposition aux perturbateurs endocriniens et l'allergie mérite d'être étudié en tenant compte de la rapide augmentation de la prévalence de l'allergie au cours des dernières décennies principalement dans les pays occidentalisés. Cette revue fait état des données s'appuyant sur l'étude de différentes cohortes reliant l'exposition des perturbateurs endocriniens, au développement de l'allergie chez l'adulte et chez le jeune enfant. Cette revue apporte également des nouvelles clés permettant d'expliciter les mécanismes aboutissant au développement de ces pathologies notamment les différentes voies de modulation de la physiologie intestinale et du dialogue système immunitaire-microbiote en lien avec la fonction endocrinienne, comme possible cible déclencheur de l'allergie.

\section{PE et maladies allergiques : données épidémiologiques}

Différentes études montrent que les PE agissent sur les êtres humains, à partir de l'âge gestationnel en passant par l'adolescence jusqu'à un âge avancé, et peuvent être considérés comme d'importants facteurs qui contribuent au développement de maladies chroniques inflammatoires, en particulier les maladies allergiques (Table 1) (13).

\subsection{Le bisphénol A}

Le Bisphénol A (BPA) est contenu dans les plastiques de polycarbonate et les résines époxydes et est utilisé dans la production de chlorure de polyvinyle (14). Les articles contenant du BPA comprennent principalement les jouets pour enfants, des récipients pour aliments et boissons tels que des bouteilles d'eau en plastique, des emballages alimentaires et le revêtement intérieur des 
canettes et des bouteilles. La principale voie d'exposition du BPA est la voie orale (15). II existe très peu d'étude épidémiologique sur l'occurrence des allergies chez l’adulte en lien avec la présence de perturbateurs endocriniens. Une enquête épidémiologique chez une population adulte décrit un potentiel lien entre l'exposition à des polluants environnementaux tel que le bisphénol $A$ et l'occurrence des maladies allergiques. De façon plus précise, une revue récente recense plusieurs études épidémiologiques démontrant une association entre une exposition importante au BPA et le développement de la respiration sifflante et d'autres symptômes respiratoires (16). Dans une étude transversale des données NHANES, Vaidya et al., montrent que les niveaux de BPA urinaires sont significativement associés à l'asthme allergique chez la femelle mais pas chez les males (17). Une deuxième étude NHANES de 2003-2006 n'a trouvé aucun lien entre des taux plus élevés de BPA et une allergie ou une rhinite allergique (18). Une étude de cohorte de 375 femmes enceintes au cours du troisième trimestre et de leurs enfants de 3, 5 et 7 ans a examiné les effets du BPA mesurés dans des échantillons d'urine sur le développement de l'asthme. Paradoxalement, un niveau prénatal plus élevé de BPA était liée à une réduction du nombre de sifflements à 5 ans. En revanche, des niveaux postnataux plus élevées de BPA à l'âge de 3 ou 7 ans étaient associées à une respiration sifflante plus fréquente. Ces résultats confirment que l'exposition continue ou postnatale au BPA augmente la probabilité de développer une respiration sifflante (19). De même, parmi une deuxième étude de cohorte portant sur 657 femmes enceintes, le risque relatif de respiration sifflante a augmenté avec chaque doublement de la concentration de BPA urinaire pour tous les groupes d'âge $(20,21)$. Un lien entre l'exposition prénatale au BPA et le risque de développer des maladies allergiques très précocement après la naissance a été mis en évidence (22). Les mères des enfants avec des maladies allergiques ont de façon significative des niveaux plus haut de BPA urinaires comparés à celles ayant

151 des enfants non allergiques (22). Ce fait peut être expliqué par la transmission transplacentaire de ce 152 contaminant. De plus, cette association est limitée au genre féminin et aux enfants dont les mères 153 sont plus jeunes que 25 ans. 

pendant les premiers et troisième trimestres ont été analysés. Les enfants ont été évalués pour la présence de respiration sifflante via un questionnaire tous les 6 mois pendant 3 ans. Comme cela a été constaté dans des études antérieures, le BPA était présent dans plus de $99 \%$ des échantillons d'urine maternels. Les mères ayant des concentrations de BPA dans l'urine supérieures à la médiane avaient des enfants plus susceptibles de signaler une respiration sifflante à l'âge de 6 mois, mais pas à 3 ans. Une analyse plus approfondie des données a montré que les concentrations plus élevées de BPA dans les urines à 16 semaines de gestation, mais pas à 26 semaines, étaient associées au développement d'une respiration sifflante (23). Dans une population essentiellement urbaine de femmes enceintes et de nouveau-nés, les concentrations maternelles de BPA n'étaient pas associées à des effets immunotoxiques se manifestant par une augmentation des taux élevés d'IgE, de TSLP ou d'IL-33 (24). De plus, on sait peu de choses sur les différences de genre dans ces associations. Une étude récente montre clairement que l'exposition au BPA est associée aux taux d'IgE et peut augmenter le risque de développer des maladies allergiques chez les enfants, en particulier chez les filles (25). En se basant sur les résultats de ces études épidémiologiques, d'un point de vue réglementaire, les experts de l'EFSA ont conclu que les associations rapportées ne fournissent pas de preuves suffisantes pour déduire un lien de causalité entre l'exposition au BPA pendant la grossesse ou l'enfance et les effets immunitaires, dont l'allergie, chez l'Homme. En conclusion, d'autres études sont urgentes pour explorer le mécanisme sous-jacent d'effet indésirable de l'exposition au BPA sur

173 I'asthme ainsi que sur l'allergie alimentaire, pour laquelle nous disposons de peu ou pas de données.

\subsection{Les Phthalates}

Les phtalates sont des diesters synthétiques : des esters d'acide phtalique-dialkyle ou alkyl

177 /aryle de l'acide 1,2-benzène dicarboxylique (26). Les structures chimiques de chaque phthalate varient principalement en fonction de leurs chaînes latérales et de leur poids moléculaire. Les 
Les phthalates de faible poids moléculaire sont couramment présents dans les cosmétiques et les produits de soins personnels, alors que les phthalates de haut poids moléculaires sont principalement associés aux plastiques, en particulier des matériaux de construction contenant le polychlorure de vinyle (PVC). Ils servent à augmenter la stabilité et flexibilité des composés. Les expositions alimentaires sont probablement la source la plus courante d'exposition, en particulier ceux de poids moléculaire élevé en raison de l'exposition à des emballages alimentaires contenant du PVC (27). Une récente méta-analyse d'études épidémiologiques effectuées chez les populations adultes allant de 1950 à 2007, basée sur 27 études toxicologiques révèlent une association entre l'exposition aux fumées de polychlorures de vinyle (PVC) et l'apparition des symptômes respiratoires (28) (Table 1). L'augmentation de la consommation de phthalates dans la fabrication de matériaux en PVC et d'autres nombreux produits de consommation a été fortement soupçonné d'être associé à la rapide augmentation de plusieurs maladies inflammatoires chroniques, y compris l'allergie chez les enfants. Dans ce but, une cohorte d'enfants en Suède (198 enfants présentant des symptômes allergiques persistants et 202 témoins) a été étudiée pour les liens entre l'exposition aux phthalates, y compris la BBzP et le DEHP (mesurés dans la poussière domestique), et le développement d'allergie et d'asthme. Des niveaux plus élevés de BBzP ont été mesurés dans la poussière domestique des cas allergiques. Une concentration plus élevée de DEHP dans la poussière était associée au diagnostic d'asthme. Sans surprise, la présence de revêtements de sol en PVC dans la maison était également associée au diagnostic d'asthme. De plus, la concentration de diéthyl-hexyl-phthalate et de phthalate bis (2-éthylhexyl) phthalate (DEHP) dans la poussière intérieure est positivement corrélée à une respiration sifflante à l'âge préscolaire $(29,30)$. Une étude cas-témoins similaire menée en Bulgarie a étudié 102 enfants souffrant de respiration sifflante, d'eczéma ou de rhinite et 82 témoins sains, tous âgés de 2 à 7 ans. Des concentrations plus élevées de DEHP ont été trouvées dans la poussière domestique des cas allergiques par rapport aux témoins. Le niveau de DEHP était lié à des épisodes de respiration sifflante au cours des 12 derniers mois et l'analyse a suggéré l'existence d'une relation dose-réponse avec l'exposition au DEHP (31). Les données de l'étude NHANES de 2005-2006 ont été 
examinées pour déterminer la relation entre les phthalates et les symptômes allergiques, notamment l'asthme, la respiration sifflante, l'allergie, la rhinite et le rhume des foins chez les adultes et les enfants (> 6 ans). Dans la population adulte, les taux de MBzP étaient associés à l'asthme, à la respiration sifflante, au rhume des foins et à la rhinite allergique. Des associations inverses ou non significatives ont été détectées chez les enfants (32). Plusieurs études prospectives ont approfondi nos connaissances sur le lien entre phthalates et les allergies. Dans l'une, le risque d'exposition prénatale aux phthalates a été examiné pour le développement des infections des voies respiratoires, des allergies ou de l'asthme. Des niveaux plus élevés de métabolites du DEHP ont été associés au développement des infections pulmonaires, de respiration sifflante et d'asthme (20). Une étude prospective récente s'appuyant sur l'étude de la cohorte mère-enfant EDEN a évalué l'association entre la concentration de métabolites du phthalate urinaire chez les mères prélevées entre la 24 et $28^{\text {ème }}$ semaine de gestation et l'occurrence de l'eczéma chez leur fils à l'âge de 5 ans. Cette étude démontre que l'occurrence de cette maladie chez les jeunes enfants peut être influencée par une exposition prénatale à certains phthalates chez les garçons (33). En utilisant cette même cohorte EDEN, une association négative entre les concentrations prénatales urinaires de 2,5dichlorophenol, d'éthyl-parabens, de bisphenol A et de DIDP et la santé respiratoire chez les garçons jusqu'à l'âge de 5 ans a été montré (21). dans une grande variété de produits de soins personnels (34). Le triclosan et les parabènes ont des propriétés antibactériennes (35) et des caractéristiques perturbant le système endocrinien (36). L'exposition à ces produits chimiques sont omniprésentes et se produisent par de multiples voies, y compris l'ingestion et l'absorption provenant d'applications cutanées ou via les muqueuses. Dans l'enquête nationale sur la santé et la nutrition (NHANES), qui a été administré aux États-Unis par les centres de contrôle et de prévention des maladies $(C D C)$, le triclosan était détectable dans l'urine 
chez près de $75 \%$ des participants et pour les parabènes de $99,1 \%, 92,7 \%, 47 \%$ et $42,4 \%$ pour les méthyl, propyl, butyl et éthyl parabènes, respectivement (37). Des études transversales suggèrent que l'exposition au triclosan et parabène peut augmenter le risque de maladie allergique. Les études utilisant les données de NHANES ont trouvé une association positive entre la concentration de triclosan et diagnostic avec le rhume des foins (18) et la sensibilisation allergique (38) chez les enfants âgés de 6 à 18 ans et avec des exacerbations récentes de l'asthme chez les asthmatiques de 6 ans et plus. Une étude d'enfants norvégiens de 10 ans a également trouvé une association entre les concentrations de triclosan urinaire et la sensibilisation allergique et la rhinite (39). Les preuves en ce qui concerne l'exposition aux parabènes a été moins cohérente. Dans l'études de NHANES, les concentrations urinaires de propyl, butyl et de méthyl parabènes étaient positivement associées à la sensibilisation aux aéroallergènes. Cependant, les concentrations en méthyl-parabène ont été négativement associées à l'asthme non-atopique ou à la respiration sifflante (40). Dans une étude récente, aucune association cohérente entre les concentrations de triclosan ou de parabène prénatal et précoce, et l'asthme infantile, la respiration sifflante ou la sensibilisation allergique dans la population globale a été montré (41).

\section{Mécanisme d'action des PE dans l'allergie}

\subsection{Implication du système immunitaire}

Une allergie est définie comme "réponse immunitaire indésirable qui se produit de manière reproductible à l'exposition à un allergène donné. L'allergie se développe à la suite d'une défaillance de la tolérance orale, un défaut de réponse immunitaire. Les perturbateurs endocriniens peuvent affecter non seulement le système endocrinien mais également le système immunitaire de diverses manières. Au niveau des organes cibles poumons ou intestins dans la majorité des cas, le BPA affecte la fonction « barrière » et l'homéostasie du système immunitaire et celui du microbiote. Au sein du tractus intestinal, la tolérance immunitaire des muqueuses vis-à-vis du contenu luminal est gouvernée par un ensemble de signaux fourni par les cellules immunitaires innées qui façonnent les 
réponses immunitaires adaptatives. L'épithélium muqueux commande ce réseau de régulation

259 immunitaire par sa fonction de barrière et par les signaux induits lors des contacts cellulaires et la 260 production de cytokines et de chimiokines. Le BPA a récemment révélé un fort potentiel perturbateur entrainant le dérèglement des réponses immunitaires, en particulier lorsque la période d'exposition débute in utero et se poursuit pendant la période néonatale. Ainsi, notre équipe a montré que l'exposition périnatale à de faibles doses de BPA $(5 \mu \mathrm{g} / \mathrm{kg} \mathrm{pc} / \mathrm{jour})$ perturbait I'homéostasie immunitaire de la muqueuse intestinale, s'accompagnant d'un défaut dans la mise en place et la maintenance de la tolérance orale chez la progéniture vis-à-vis d’un antigène alimentaire $(42,43)$ En effet, les souris adultes exposées via la mère à une faible dose de BPA $(50 \mu \mathrm{g} / \mathrm{kg}$ de $\mathrm{pc/jour)} \mathrm{ont} \mathrm{une} \mathrm{altération} \mathrm{de} \mathrm{la} \mathrm{barrière} \mathrm{intestinale} \mathrm{avec} \mathrm{une} \mathrm{production} \mathrm{réduite} \mathrm{d'acide} \mathrm{rétinoïque}$ par les cellules épithéliales intestinales (IEC). De plus, la production des IgA et des IgG totaux, dans 269 les fèces, est également réduite. Chez la descendance, on observe une augmentation de la 270 perméabilité colique associée avec l'augmentation de l'interféron-gamma et une chute des cellules $\lg \mathrm{A}^{+}$coliques et de la production d'IgA fécale (44). Ces altérations au niveau de la barrière épithéliale intestinale sont associées à un défaut de maturation des cellules dendritiques de la LP et de la rate.

273 Ces résultats montrent que l'exposition périnatale au BPA affaiblit les fonctions protectrices et régulatrices des cellules épithéliales intestinales associées à une altération de la maturation des cellules dendritiques (DC) et une différenciation inappropriée des cellules T et ILC3. Ces perturbations conduisent à une détérioration des réponses tolérogènes en faveur des réponses immunitaires

277 systémiques inflammatoires chez la descendance, pouvant ainsi expliquer le défaut de tolérance 278 orale chez ces animaux suite à une exposition périnatale à ce perturbateur endocrinien (44) (Figure 279 1). De plus, l'exposition au BPA peut moduler la réaction allergique. En effet, le bisphénol A 280 augmente la sécrétion d'IL-4 par la lymphocyte CD4 de façon dose dépendante chez les souris 281 sensibilisées $(33,34)$. Ces résultats suggèrent que le BPA peut augmenter la réponse IgE médiée dans 282 I'allergie via l'augmentation de la production d'IL-4. Ainsi, l'exposition in vivo au BPA donne lieu à augmentation des taux d'IgE spécifiques de l'antigène dans les sérums de souris allergiques (34). Cela 
supporte I'hypothèse que le BPA possède la capacité d'exacerber l'allergie (4) (Figure 1). II a été proposé que les conditions où le BPA provoque une prédominance des cellules Th2 favorise le développement de l'allergie ou l'asthme $(36,37)$. Nous et d'autres avons démontré que l'exposition gestationnelle et lactationnelle des souris au BPA entraîne une augmentation du nombre de cellules Th17 $(26,38)$. Enfin, nos travaux ont été montré que l'exposition maternelle au BPA peut avoir un impact sur la fonction des lymphocytes T régulateurs (Tregs), cellules qui sont connues pour contenir et contrôler les réponses immunitaires $(26,39)$.

\subsection{Implication du microbiote}

Dans l'allergie, la flore microbienne au niveau pulmonaire et intestinal joue également un rôle prépondérant mais encore mal défini. Le microbiote de la sphère intestinale a été plus étudié que le celui retrouvé au niveau pulmonaire dans l'allergie. Des changements dans ce dernier conduit à des changements dans les métabolites bactériens (comme la chaîne courte des acides gras) essentiels au maintien de l'intégrité des muqueuses et à la promotion de la tolérance orale. Des preuves limitées démontrent l'altération du microbiote intestinal chez les enfants présentant une allergie alimentaire. Dans un modèle de souris gnotobiotique, la colonisation sélective de l'intestin avec le microbiote contenant Clostridia protège de l'allergie alimentaire via l'activation des cellules lymphoïdes innées, productrices d'IL-22 et l'amélioration de la perméabilité intestinale (45). Plusieurs études récentes montrent que le BPA affecte le microbiote intestinal aboutissant à une dysbiose. Une première étude a comparé le microbiote de souris mâles âgées de 21 jours après exposition en direct au BPA (eau de boisson, $120 \mu \mathrm{g} / \mathrm{mL}$ ) pendant 70 jours avec celui de souris nourries à un régime riche en graisse (HFD pour High-Fat Diet) ou en sucre (HSD pour High-Sucrose Diet) (46). Les résultats ont montré une diminution significative de la diversité du microbiote intestinal des individus exposés au BPA. De plus, l'exposition au BPA mène à une dysbiose similaire à celui d'un régime HFD ou HSD. Une deuxième étude a évalué les conséquences sur le microbiote intestinal de l'exposition périnatale au BPA à $50 \mathrm{mg} / \mathrm{kg}$ de nourriture deux semaines avant accouplement et jusqu'au jour 30 post natal, à la fois chez la descendance (âgée de 30 jours) et chez les parents (47). Les résultats ont montré des 
changements générationnels et sexe-dépendants du microbiote intestinal. De manière intéressante,

311 les Bifidobacteria, bénéfiques pour l'intestin, sont retrouvés augmentées seulement chez la descendance femelle. L'exposition au BPA provoque donc une dysbiose intestinale qui pourrait contribuer à la sensibilisation ou le déclenchement de réactions allergiques. Le mécanisme du BPA sur ses effets modulateurs de microbiote intestinal fait encore débat et il n'existe pas de données sur le lien entre effets du BPA sur le microbiote pulmonaire et asthme. Cependant, étant donné le lien étroit entre le système immunitaire et le microbiote intestinal, les dysbioses observées après exposition au BPA peuvent également être soit la cause soit la conséquence de l'altération du système immunitaire intestinal aboutissant au défaut de tolérance orale (48-51). connus pour contribuer à l'incidence des allergies chez les femmes (52), ce qui suggère un rôle pour les œstrogènes et d'autres hormones sur l'inflammation allergique. De plus, l'incidence des pics d'allergie chez les femmes atteint un seuil après la puberté et diminue fortement avec l'âge. 33-52\% des femmes asthmatiques signalent une aggravation prémenstruelle de leurs symptômes. apparaissent relevant dans la pathogénèse de l'asthme. Dans l'asthme, les macrophages alvéolaires ont émergé en tant que médiateurs cellulaires importants de l'inflammation et du remodelage tissulaire. L'asthme allergique est considéré comme une maladie Th2 et est donc accompagnée de macrophages alvéolaires M2 polarisés en IL-4/13 dans les voies respiratoires. Les macrophages dérivés de la moelle osseuse (BMM) ont servi de modèle de substitution in vitro pour des études portant sur l'effet de l'œstrogène sur la biologie des macrophages. La polarisation M2 induite par IL-4 est renforcée par l'addition d'œstrogène exogène à des doses micromolaires dans des BMM de souris (53). Les DCs sont des initiateurs essentiels des réponses immunitaires adaptatives qui facilitent la sensibilisation aux allergènes. L'œstrogène est étroitement impliqué dans la fonction des 
DCs, de la différenciation à la maturation et la production de cytokines pro-inflammatoires. Comme

337 les macrophages, des études suggèrent que les œstrogènes ont des effets sur les DCs en augmentant

338 la production de cytokines inflammatoires en réponse au ligand du TLR et en favorisant l'activation

339 des lymphocytes T. Ces deux mécanismes étant lié de façon étroite au développement des allergies.

340 Les effets de l'œstrogène sur les cellules T sont complexes et varient en fonction le contexte et la

341 dose. Aux concentrations retrouvées chez la femmes pendant la grossesse (1-150 nM), l'œstrogène

342 favorise la production d'IFNY, de FoxP3 et CD25 par les lymphocytes T de souris in vivo et in vitro

343 d'une manière dépendante de ER $\alpha$ (54). De façon général, l'œstrogène améliore la maturation des

344 lymphocytes B et la production des anticorps (55). De plus, de fortes concentrations d'œstrogènes

345 (gamme micromolaire) augmentent la quantité d'anticorps produite par des cellules mononucléées

346 du sang périphérique de souris stimulées par un mitogène (56). Surtout, les œstrogènes et les

347 composés œstrogéniques augmentent la production d'IgE splénocytes de souris et peuvent ainsi

348 contribuer à l'inflammation allergique (57). Les EDC sont des produits chimiques exogènes capables

349 d'interférer avec le système endocrinien endogène.

350 En effet, il a été démontré que les alkylphénols, un groupe répandu de PE, se lient aux

351 récepteurs des œstrogènes (ER) (58) et s'accumulent dans l'organisme en raison de leurs propriétés

352 lipophiles et de leurs longues demi-vies. Étant donné que les œstrogènes jouent un rôle important

353 dans le développement et la fonction des cellules immunitaires, les alkylphénols pourraient

354 perturber la régulation immunitaire par leurs actions sur les cellules immunitaires exprimant les ER.

355 Ainsi, les alkylphénols peuvent modifier les réponses immunitaires car les œstrogènes sont capables

356 de réguler les fonctions des cellules immunitaires, telles que les cellules dendritiques (DC) et les

357 cellules T $(59,60)$. L'accumulation de données in vitro suggère que les alkylphénols peuvent favoriser

358 les réactions allergiques, en partie à travers les ER.

359 D'autre part, le BPA est connu pour être un xénoestrogène car il interagit avec les récepteurs

360 aux œstrogènes (ER). II agit comme agoniste ou antagoniste via des voies de signalisation

361 dépendantes du ER. La cinétique de la liaison du BPA aux ERs a montré que le BPA se lie aux deux 
$E R \alpha$ et $E R ß$, avec une affinité environ 10 fois supérieure à $E R ß ~(42,43)$. L'effet de ce PE décrit précédemment sur le système immunitaire peut s'expliquer à travers son interaction avec les ER. La contribution respective de ER $\alpha, E R \beta$ et GPR-1 dans les fonctions immunitaires innées et adaptatives de l'inflammation allergique a besoin d'être exploré en utilisant les souris knockout cibles ou inductibles in vivo (61).

\section{Conclusion}

Il est tout à fait légitime d'explorer si les produits chimiques auxquels les humains sont exposés peuvent altérer ou perturber le système immunitaire d'une manière susceptible d'accroître les risques néfastes sur la santé. Il est donc approprié de considérer le potentiel du BPA et des autres PE à potentialiser ou à prédisposer à une maladie allergique.

Les preuves examinées dans cette revue proviennent d'études humaines, d'investigations sur des modèles animaux et d'expériences in vitro. Plusieurs études démontrent que le BPA peut affecter le système immunitaire et son développement. Cependant, il n'existe actuellement aucun consensus convaincant démontrant l'influence du BPA et des autres PE, sur les allergies. Les recherches futures devront examiner le mécanisme d'action de ces produits chimiques et la relation dose-effet. Il sera essentiel de déterminer la période clé d'exposition à ces produits chimiques et leurs effets à long terme, ainsi que les populations à risque. De plus, si l'existence d'une relation entre ces substances chimiques et le développement des allergies est confirmée, il sera nécessaire de déterminer des méthodes préventives pour limiter leurs impacts.

\section{Remerciement}

Ce travail a été soutenu par la subvention EST-2015/1/026 de I'Agence Nationale de Sécurité Sanitaire, de l'Alimentation, de l'Environnement et du Travail (ANSES). 
388 1. Foong RX, du Toit G, Fox AT. Asthma, Food Allergy, and How They Relate to Each Other. 389 Frontiers in pediatrics. 2017;5:89.

$390 \quad$ 2. Fox A, du Toit G, Foong RX. Mini Review - Asthma and food allergy. Current pediatric reviews. 3912018.

3. Berin MC, Sampson HA. Mucosal immunology of food allergy. Current biology : CB. 2013;23(9):R389-400.

4. Kalach N, Rocchiccioli F, de Boissieu D, Benhamou PH, Dupont C. Intestinal permeability in children: variation with age and reliability in the diagnosis of cow's milk allergy. Acta paediatrica. 2001;90(5):499-504.

5. Chahine BG, Bahna SL. The role of the gut mucosal immunity in the development of tolerance versus development of allergy to food. Current opinion in allergy and clinical immunology. 2010;10(4):394-9.

400

6. Kanny G. [Food allergy]. La Revue du praticien. 2007;57(12):1331-8.

7. Mastrorilli C, Caffarelli C, Hoffmann-Sommergruber K. Food allergy and atopic dermatitis: Prediction, progression, and prevention. Pediatric allergy and immunology : official publication of the European Society of Pediatric Allergy and Immunology. 2017;28(8):831-40.

8. Dietert RR. Fractal immunology and immune patterning: potential tools for immune protection and optimization. Journal of immunotoxicology. 2011;8(2):101-10.

9. Heindel JJ. A special Issue on the environment and Developmental Origins of Health and Diseases. Reproductive toxicology. 2017;68:1-2.

10. Chalubinski M, Kowalski ML. Endocrine disrupters--potential modulators of the immune 409 system and allergic response. Allergy. 2006;61(11):1326-35.

410 11. Schug TT, Janesick A, Blumberg B, Heindel JJ. Endocrine disrupting chemicals and disease 411 susceptibility. The Journal of steroid biochemistry and molecular biology. 2011;127(3-5):204-15. 
413 European Commission document, "State of the Art Assessment of Endocrine Disrupters". Critical 414 reviews in toxicology. 2012;42(6):465-73.

13. Kuo $\mathrm{CH}$, Yang SN, Kuo PL, Hung $\mathrm{CH}$. Immunomodulatory effects of environmental endocrine disrupting chemicals. The Kaohsiung journal of medical sciences. 2012;28(7 Suppl):S37-42.

417 14. Rosenfeld CS. Bisphenol A and phthalate endocrine disruption of parental and social behaviors. Frontiers in neuroscience. 2015;9:57.

15. Vandenberg LN, Hauser R, Marcus M, Olea N, Welshons WV. Human exposure to bisphenol A (BPA). Reproductive toxicology. 2007;24(2):139-77.

16. Robinson L, Miller R. The Impact of Bisphenol A and Phthalates on Allergy, Asthma, and Immune Function: a Review of Latest Findings. Current environmental health reports. 2015;2(4):37987.

17. Vaidya SV, Kulkarni H. Association of urinary bisphenol A concentration with allergic asthma: results from the National Health and Nutrition Examination Survey 2005-2006. The Journal of asthma : official journal of the Association for the Care of Asthma. 2012;49(8):800-6. parameters in the U.S. population, NHANES 2003-2006. Environmental health perspectives. 2011;119(3):390-6.

19. Donohue KM, Miller RL, Perzanowski MS, Just AC, Hoepner LA, Arunajadai S, et al. Prenatal and postnatal bisphenol $\mathrm{A}$ exposure and asthma development among inner-city children. The Journal of allergy and clinical immunology. 2013;131(3):736-42. 
437 Exposure to Select Phenols and Phthalates and Respiratory Health in Five-Year-Old Boys: A 438 Prospective Study. Environmental health perspectives. 2017;125(9):097006.

22. Zhou A, Chang H, Huo W, Zhang B, Hu J, Xia W, et al. Prenatal exposure to bisphenol A and risk of allergic diseases in early life. Pediatric research. 2017;81(6):851-6.

23. Spanier AJ, Kahn RS, Kunselman AR, Hornung R, Xu Y, Calafat AM, et al. Prenatal exposure to bisphenol $A$ and child wheeze from birth to 3 years of age. Environmental health perspectives. 2012;120(6):916-20.

24. Ashley-Martin J, Dodds L, Levy AR, Platt RW, Marshall JS, Arbuckle TE. Prenatal exposure to phthalates, bisphenol A and perfluoroalkyl substances and cord blood levels of IgE, TSLP and IL-33. Environmental research. 2015;140:360-8.

25. Wang IJ, Chen CY, Bornehag CG. Bisphenol A exposure may increase the risk of development 448 of atopic disorders in children. International journal of hygiene and environmental health. $449 \quad$ 2016;219(3):311-6.

450 26. North ML, Takaro TK, Diamond ML, Ellis AK. Effects of phthalates on the development and 451 expression of allergic disease and asthma. Annals of allergy, asthma \& immunology : official 452 publication of the American College of Allergy, Asthma, \& Immunology. 2014;112(6):496-502.

453 27. Buckley JP, Palmieri RT, Matuszewski JM, Herring AH, Baird DD, Hartmann KE, et al. 454 Consumer product exposures associated with urinary phthalate levels in pregnant women. Journal of 455 exposure science \& environmental epidemiology. 2012;22(5):468-75.

45628 . Jaakkola JJ, Knight TL. The role of exposure to phthalates from polyvinyl chloride products in 457 the development of asthma and allergies: a systematic review and meta-analysis. Environmental 458 health perspectives. 2008;116(7):845-53.

459 29. Kolarik B, Lagercrantz L, Sundell J. Nitric oxide in exhaled and aspirated nasal air as an 460 objective measure of human response to indoor air pollution. Indoor air. 2009;19(2):145-52. 

andrology. 2010;33(2):333-45.

463 31. Kolarik B, Naydenov K, Larsson M, Bornehag CG, Sundell J. The association between 464 phthalates in dust and allergic diseases among Bulgarian children. Environmental health

465 perspectives. 2008;116(1):98-103.

32. Hoppin JA, Jaramillo R, London SJ, Bertelsen RJ, Salo PM, Sandler DP, et al. Phthalate exposure and allergy in the U.S. population: results from NHANES 2005-2006. Environmental health perspectives. 2013;121(10):1129-34 .

33. Soomro MH, Baiz N, Philippat C, Vernet C, Siroux V, Nichole Maesano C, et al. Prenatal Exposure to Phthalates and the Development of Eczema Phenotypes in Male Children: Results from the EDEN Mother-Child Cohort Study. Environmental health perspectives. 2018;126(2):027002.

34. Calafat AM, Weuve J, Ye X, Jia LT, Hu H, Ringer S, et al. Exposure to bisphenol A and other phenols in neonatal intensive care unit premature infants. Environmental health perspectives. 2009;117(4):639-44.

35. Bredin J, Davin-Regli A, Pages JM. Propyl paraben induces potassium efflux in Escherichia coli. The Journal of antimicrobial chemotherapy. 2005;55(6):1013-5.

36. Witorsch RJ, Thomas JA. Personal care products and endocrine disruption: A critical review of the literature. Critical reviews in toxicology. 2010;40 Suppl 3:1-30.

37. Calafat AM, Ye X, Wong LY, Bishop AM, Needham LL. Urinary concentrations of four parabens in the U.S. population: NHANES 2005-2006. Environmental health perspectives. 2010;118(5):679-85. 38. Spanier AJ, Kahn RS, Kunselman AR, Schaefer EW, Hornung $R, X u Y$, et al. Bisphenol a exposure and the development of wheeze and lung function in children through age 5 years. JAMA pediatrics. 2014;168(12):1131-7.

39. Bertelsen RJ, Carlsen KC, Calafat AM, Hoppin JA, Haland G, Mowinckel P, et al. Urinary biomarkers for phthalates associated with asthma in Norwegian children. Environmental health perspectives. 2013;121(2):251-6. 
40. Savage JH, Matsui EC, Wood RA, Keet CA. Urinary levels of triclosan and parabens are

488

489

490

491

492

493

494

495

496

497

498

499

500

501

502

503

504

505

506

507

508

509

510

associated with aeroallergen and food sensitization. The Journal of allergy and clinical immunology. 2012;130(2):453-60 e7.

41. Lee-Sarwar K, Hauser R, Calafat AM, Ye X, O'Connor GT, Sandel M, et al. Prenatal and earlylife triclosan and paraben exposure and allergic outcomes. The Journal of allergy and clinical immunology. 2018;142(1):269-78 e15.

42. Menard S, Guzylack-Piriou L, Leveque M, Braniste V, Lencina C, Naturel M, et al. Food intolerance at adulthood after perinatal exposure to the endocrine disruptor bisphenol A. FASEB journal : official publication of the Federation of American Societies for Experimental Biology. 2014;28(11):4893-900.

43. Menard S, Guzylack-Piriou L, Lencina C, Leveque M, Naturel M, Sekkal S, et al. Perinatal exposure to a low dose of bisphenol A impaired systemic cellular immune response and predisposes young rats to intestinal parasitic infection. PloS one. 2014;9(11):e112752.

44. Malaise Y, Menard S, Cartier C, Lencina C, Sommer C, Gaultier E, et al. Consequences of bisphenol a perinatal exposure on immune responses and gut barrier function in mice. Archives of toxicology. 2018;92(1):347-58.

45. Stefka AT, Feehley T, Tripathi P, Qiu J, McCoy K, Mazmanian SK, et al. Commensal bacteria protect against food allergen sensitization. Proceedings of the National Academy of Sciences of the United States of America. 2014;111(36):13145-50.

46. Lai KP, Chung YT, Li R, Wan HT, Wong CK. Bisphenol A alters gut microbiome: Comparative metagenomics analysis. Environmental pollution. 2016;218:923-30.

47. Javurek AB, Spollen WG, Johnson SA, Bivens NJ, Bromert KH, Givan SA, et al. Effects of exposure to bisphenol $A$ and ethinyl estradiol on the gut microbiota of parents and their offspring in a rodent model. Gut microbes. 2016;7(6):471-85.

48. Palm NW, de Zoete MR, Flavell RA. Immune-microbiota interactions in health and disease.

Clinical immunology. 2015;159(2):122-7. 
514 mediator of gut homeostasis. Cell host \& microbe. 2007;2(5):328-39.

515 50. Reddivari L, Veeramachaneni DNR, Walters WA, Lozupone C, Palmer J, Hewage MKK, et al. Perinatal Bisphenol A Exposure Induces Chronic Inflammation in Rabbit Offspring via Modulation of Gut Bacteria and Their Metabolites. mSystems. 2017;2(5).

518 51. Chen L, Guo Y, Hu C, Lam PKS, Lam JCW, Zhou B. Dysbiosis of gut microbiota by chronic coexposure to titanium dioxide nanoparticles and bisphenol A: Implications for host health in zebrafish. Environmental pollution. 2018;234:307-17.

52. Yung JA, Fuseini H, Newcomb DC. Hormones, sex, and asthma. Annals of allergy, asthma \& immunology : official publication of the American College of Allergy, Asthma, \& Immunology. 2018;120(5):488-94.

53. Campbell L, Emmerson E, Williams H, Saville CR, Krust A, Chambon P, et al. Estrogen receptor-alpha promotes alternative macrophage activation during cutaneous repair. The Journal of investigative dermatology. 2014;134(9):2447-57.

54. Polanczyk M, Yellayi S, Zamora A, Subramanian S, Tovey M, Vandenbark AA, et al. Estrogen receptor-1 (Esr1) and -2 (Esr2) regulate the severity of clinical experimental allergic encephalomyelitis in male mice. The American journal of pathology. 2004;164(6):1915-24.

55. Kanda R, Hayata I. Effect of estradiol on radiation-induced chromosome aberrations in human lymphocytes. Journal of radiation research. 1999;40(2):95-100. response. Estradiol enhances human B cell maturation via inhibition of suppressor $\mathrm{T}$ cells in pokeweed mitogen-stimulated cultures. The Journal of experimental medicine. 1981;154(6):1935-45. hydrogenated and saturated, relative to polyunsaturated, fat on immune and inflammatory 537 responses of adults with moderate hypercholesterolemia. Journal of lipid research. 2002;43(3):44552. 
58. Kuiper GG, Lemmen JG, Carlsson B, Corton JC, Safe SH, van der Saag PT, et al. Interaction of

540 estrogenic chemicals and phytoestrogens with estrogen receptor beta. Endocrinology. 1998;139(10):4252-63.

59. Carreras E, Turner S, Paharkova-Vatchkova V, Mao A, Dascher C, Kovats S. Estradiol acts directly on bone marrow myeloid progenitors to differentially regulate GM-CSF or Flt3 ligandmediated dendritic cell differentiation. Journal of immunology. 2008;180(2):727-38.

60. Michalek RD, Gerriets VA, Nichols AG, Inoue M, Kazmin D, Chang CY, et al. Estrogen-related receptor-alpha is a metabolic regulator of effector T-cell activation and differentiation. Proceedings of the National Academy of Sciences of the United States of America. 2011;108(45):18348-53.

61. Keselman A, Heller N. Estrogen Signaling Modulates Allergic Inflammation and Contributes to

Figure 1: Modulation de la réponse immunitaire pulmonaire et intestinale par les perturbateurs populations les plus sensibles. 


\begin{tabular}{|c|c|c|c|c|c|}
\hline $\begin{array}{l}\text { Nombre de } \\
\text { participants }\end{array}$ & Conception de l'étude & Mesure & Age & Résultat/observation(s) & Citation \\
\hline \multicolumn{6}{|l|}{ Bisphénol A } \\
\hline 5250 & $\begin{array}{l}\text { Etude transversale (NHANES 2005- } \\
\text { 2006) }\end{array}$ & BPA urinaire & Adultes et enfants $>6$ ans & Pas d'association + avec allergie & Clayton et al., 2011 \\
\hline 365 & Etude prospective & BPA urinaire & $2 \mathrm{e}$ et $3 \mathrm{eme}$ trimestre de gestation & Respiration sifflante at 6 mois & Spanier, 2012 \\
\hline 2548 & $\begin{array}{l}\text { Etude transversale (NHANES 2005- } \\
\text { 2006) }\end{array}$ & BPA urinaire & Adultes : 1270 males/1278 femelles & $\begin{array}{l}\text { association significative avec asthme allergique } \\
\text { chez la femelle }\end{array}$ & Vaidya, 2012 \\
\hline 375 & Etude prospective & BPA urinaire & $\begin{array}{l}\text { 3eme trimestre de gestation, enfant de } 3,5,7 \\
\text { ans }\end{array}$ & $\begin{array}{l}\text { Concentration de BPA en prénatal inversement } \\
\text { associée à une respiration sifflante à lâge de } 3 \text { ans } \\
\text { Concentration de BPA dans l'enfance positivement } \\
\text { avec respiration sifflante }\end{array}$ & Donohue, 2013 \\
\hline 661 & $\begin{array}{l}\text { Etude transversale (NHANES 2007- } \\
\text { 2010) }\end{array}$ & BPA urinaire & Enfants de $16-19$ ans & Diminution des fonctions pulmonaires & $\begin{array}{l}\text { Spanier, Fiorino, } \\
\text { Transade } 2014\end{array}$ \\
\hline 244 & Etude prospective & BPA urinaire & $\begin{array}{l}\text { 3eme trimestre de gestation, enfant de 3,5,7 } \\
\text { ans }\end{array}$ & Asthme & Whyatt 2014 \\
\hline 1258 & Etude transversale (MIREC, 2001) & $\begin{array}{l}\text { BPA urine maternelle/BPA plasma de } \\
\text { cordon ombilical }\end{array}$ & 1er trimestre de grossesse & Pas d'association avec IgE, TSLP et IL-33 & Ashley-Martin, 2015 \\
\hline 275 & Etude prospective & BPA serum & Neonates & $\begin{array}{l}\text { association significative avec niveau de TNFa } \\
\text { associé au TLR3 et TLR4 }\end{array}$ & Liao et al. 2016 \\
\hline 657 & Etude prospective & BPA urinaire & 1er et 3eme trimestre de gestation & $\begin{array}{l}\text { Respiration sifflante, asthme, infections } \\
\text { respiratoires }\end{array}$ & Gascon,2015 \\
\hline 412 & Etude prospective & BPA urinaire & $\begin{array}{l}\text { A la délivrance chez la mère, enfant de } 6 \\
\text { mois }\end{array}$ & $\begin{array}{l}\text { Maladie allergique (Respiration sifflante et } \\
\text { eczéma), specifiquement chez les filles }\end{array}$ & Zhou, 2017 \\
\hline 587 & Etude prospective (cohorte EDEN) & BPA urinaire & $\begin{array}{l}\text { Suivi de la santé respiratoire des fils jusqu'à } \\
5 \text { ans }\end{array}$ & Altération de la santé respiratoire & Vernet et al., 2017 \\
\hline \multicolumn{6}{|l|}{ Phthalates } \\
\hline 27 études toxicologiques & Méta-analyses bibliographiques & 14 laboratoires & Adultes & $\begin{array}{l}\text { Fummées de PVC contribue au développement de } \\
\text { l'asthme chez l'adulte }\end{array}$ & Jaakkola, 2008 \\
\hline 405 & Etude de cas & BBzP et DEHP & Enfants 3-8 ans & $\begin{array}{l}\text { BBzP, PVC du sol associés avec allergie, DEHP } \\
\text { avec asthme }\end{array}$ & Bornehag, 2004 \\
\hline 184 & Etude de cas & DEHP dans la poussière domestique & Enfants de 2, 3, 5 et 7 ans & Allergie & Kolarik, 2008 \\
\hline 2325 & $\begin{array}{l}\text { Etude transversale (NHANES 2005- } \\
\text { 2006) }\end{array}$ & Phthalates urinaires & Enfants de + de 6 ans, adultes & $\begin{array}{l}\text { MBzP et DEHP associés avec l'allergie chez } \\
\text { I'adultes, pas chez les enfants }\end{array}$ & Hoppin, 2013 \\
\hline 244 & Etude prospective & Phthalates urinaires prenatal & $\begin{array}{l}\text { 3eme trimestre de gestation, enfant de 3,5,7 } \\
\text { ans }\end{array}$ & Asthme & Whyatt 2014 \\
\hline 1258 & Etude transversale (MIREC, 2001) & $\begin{array}{l}\text { Urine maternelle/plasma de cordon } \\
\text { ombilical }\end{array}$ & 1er trimestre de grossesse & Pas d'association avec IgE, TSLP et IL-33 & Ashley-Martin, 2015 \\
\hline 998 & Etude prospective (cohorte EDEN) & Phthalates urinaires & $\begin{array}{l}\text { Prélèvement chez la mère entre le 24-28 } \\
\text { sem de gestation. Suivi de la descendance } \\
\text { male jusqu'à } 5 \text { ans }\end{array}$ & $\begin{array}{l}\text { occurrence de l'eczema est associé avec exposition } \\
\text { prénatal de phthalates chez les garçons }\end{array}$ & Soomro et al., 2018 \\
\hline \multicolumn{6}{|l|}{ Triclosan } \\
\hline 5250 & $\begin{array}{l}\text { Etude transversale (NHANES 2005- } \\
\text { 2006) }\end{array}$ & Triclosan urinaire & Adultes et enfants $>6$ ans & Association + avec allergie & Clayton et al., 2011 \\
\hline 860 & $\begin{array}{l}\text { Etude transversale (NHANES 2005- } \\
\text { 2006) }\end{array}$ & Triclosan urinaire & Enfants de 6 à 18 ans & Pas d'association avec sensibilisation allergique & Savage, 2012 \\
\hline 623 & Etude transversale $\quad$ (2001-2004) & Triclosan urinaire & Enfants 10 ans & Association avec senbilisation allergique et rhinites & Bertelsen , 2013 \\
\hline 837 & $\begin{array}{l}\text { Etude transversale (NHANES 2005- } \\
\text { 2006) }\end{array}$ & Triclosan urinaire & Enfants de 6 à 18 ans & Association + avec Allergie & Spanier et al., 2014 \\
\hline 860 & $\begin{array}{l}\text { Etude transversale (NHANES 2005- } \\
\text { 2006) }\end{array}$ & Triclosan urinaire & Enfants $>16$ ans & Exacerbation de l'asthme & Savage et al., 2014 \\
\hline 467 & Etude prospective & Triclosan urinaire & Enfants de 3 et 4 ans & $\begin{array}{l}\text { Pas d'association avec asthme ou sensbilisation } \\
\text { alimentaire }\end{array}$ & Lee-Sarwar et al., 2017 \\
\hline \multicolumn{6}{|l|}{ Parabens } \\
\hline 860 & $\begin{array}{l}\text { Etude transversale (NHANES 2005- } \\
\text { 2006) }\end{array}$ & Triclosan urinaire & Enfants de 6 à 18 ans & Pas d'association avec sensibilisation allergique & Savage, 2012 \\
\hline 837 & $\begin{array}{l}\text { Etude transversale (NHANES 2005- } \\
\text { 2006) }\end{array}$ & Parabens urinaire & Enfants de 6 à 18 ans & Association + avec asthme & Spanier et al., 2014 \\
\hline 467 & Etude prospective & Triclosan urinaire & Enfants de 3 et 4 ans & $\begin{array}{l}\text { Pas d'association avec asthme ou sensbilisation } \\
\text { alimentaire }\end{array}$ & Lee-Sarwar et al., 2017 \\
\hline 587 & Etude prospective (cohorte EDEN) & BPA, ethyl-parabens urinaire & $\begin{array}{l}\text { Suivi de la santé respiratoire des fils jusqu'à } \\
5 \text { ans }\end{array}$ & Altération de la santé respiratoire & Vernet et al., 2017 \\
\hline
\end{tabular}

